\title{
Effects of stimulus position in the respiratory cycle on the evoked cardiac response
}

\author{
GRAHAM TURPIN and GUDRUN SARTORY \\ Institute of Psychiatry, London SE5 8AF, England
}

\begin{abstract}
The present experiment investigated the effects of stimulus position within the respiratory cycle on the evoked cardiac response (ECR). Two independent groups of subjects $(\mathrm{N}=14)$ received six presentations of a $75-\mathrm{dB}$ tone of $1 \mathrm{sec}$ duration and instantaneous rise time. The mean interstimulus interval was $45 \mathrm{sec}$. In one group, stimuli were presented at midinspiration, whereas in the other group stimuli were delivered during midexpiration. Heart rate (HR), skin conductance responses (SCR), and respiration were measured. Stimuli presented during midinspiration produced cardiac acceleration, whereas stimuli presented during midexpiration resulted in deceleration. These differences were substantially reduced following correction for respiratory sinus arrhythmia. The corrected ECR consisted solely of cardiac deceleration in both groups. Deceleration was largest in the inspiration group. No differences in SCRs were found. These results are discussed in terms of the "vagal gating" hypothesis.
\end{abstract}

The purpose of this experiment was to investigate the effect of the interaction between respiratory and cardiac activity on the evoked cardiac response (ECR). Two aspects of cardiac-respiratory coupling were examined.

The first concerned the influence of sinus arrhythmia (SA) ${ }^{1}$ upon the quantification of the ECR. This problem has recently been reviewed and a number of techniques said to correct for the influence of SA upon the ECR have been discussed (Lobstein, Webb, \& Cort, 1978; Turpin, Lobstein, \& Siddle, 1980; Turpin \& Siddle, 1978). However, these techniques rely on the assumption that cardiac responses summate with SA in an additive fashion (Turpin \& Siddle, 1978). This assumption was first examined by Berg and Beebe-Center (1941), who suggested two alternative hypotheses regarding the interaction of respiratory sinus arrhythmia (RSA) ${ }^{1}$ and the form of the ECR. These were either that the cardiac response represents a summation of the neural activity associated with stimulus presentation and the ongoing activity present in the form of RSA or that the stimulus-evoked changes suppress and replace any activity associated with RSA. These two hypotheses were tested by comparing the ECRs elicited by a variety of intense "startle" stimuli presented at different phases of the breathing cycle. Essentially, if the summation hypothesis was correct, then the ECR would be dependent upon the ongoing pattern of RSA and hence the

This research was supported by the Medical Research Council. We are grateful to $\mathrm{L}$. Law for the use of the SARA program package for the analysis of psychophysiological data. Requests for reprints should be addressed to Graham Turpin, Institute of Psychiatry, De Crespigny Park, Denmark Hill, London SE5 8AF, England. position of the stimulus in the breathing cycle. If the replacement hypothesis was correct, stimulus position would have no effect upon the ECR. They interpreted their results in favor of the replacement hypothesis. However, the graphs that they presented were equivocal and could have easily been used in support of the summation hypothesis. Unfortunately, the results were not subjected to any form of statistical analysis, and hence the study must be considered inconclusive. Furthermore, they employed a pistol shot as the eliciting stimulus, and it is conceivable that such an intense startle stimulus may have produced a respiratory gasp that would have been independent of stimulus position in the breathing cycle and hence would favor the replacement hypothesis. This possibility is supported by the observation that the mean cardiac acceleration exceeded $60 \mathrm{bpm}$ in the expiration group. Unfortunately, respiratory activity was not analyzed in this study.

In a similar study, Hart (1975) found that the shape of the ECR was dependent upon the position of the stimulus within the breathing cycle when the ECR was expressed as a difference score from the prestimulus level. Moreover, Hart demonstrated that these differences disappear if a correction for RSA is made. This finding has been replicated by Kamath (Note 1), using the SA-corrected technique (Turpin \& Siddle, 1978). Results from these two studies support the summation hypothesis and the use of SA correction procedures in the quantification of the ECR. However, none of the studies mentioned has employed stimuli of moderate intensity which produce decelerative responses (Graham \& Clifton, 1966). The stimuli used in Hart's study consisted of 100-dB tones which elicited cardiac acceleration. Since many studies are concerned with the cardiac component of the orienting 
response (OR), this experiment will specifically examine the viability of the summation hypothesis for decelerative responses.

The second aspect of cardiac-respiratory coupling examined by this study concerns the notion of vagal gating. Hayes (Note 2) has suggested that the gain of the cardiac response mechanism is sensitive to the phase of respiration. More specifically, he has argued that the activity of vagal efferents is inhibited during inspiration. This argument is based upon a series of observations showing modulation of efferent vagal activity occurring in phase with the breathing cycle (Iriuchijima \& Kumada, 1964; Katona, Poitras, Barnett, \& Terry, 1970). Furthermore, he suggests that the inspiratory cardiac accelerative component of RSA is also mediated via vagal inhibition. Recent evidence would seem to support this notion of respiratory-linked modulation of the "vasomotor center" (Davidson, Goldner, \& McCloskey, 1976; Melcher, 1976). If this is the case, and if the cardiac decelerative ECR is assumed to be vagally mediated (Obrist, Wood, \& Perez-Reyes, 1965), it can be expected that respiratory phase will influence the form of the ECR by blocking vagal activity during inspiration. Furthermore, this effect will be independent of prestimulus variability.

The possibility of phase-related differences in the ECR which cannot be corrected by using RSA techniques is important for two reasons. First, the position of stimulation in the breathing cycle should be seen as an important variable in studies of the ECR; if uncontrolled, it may account for a large proportion of the error variance. Second, the differential occurrence of cardiac deceleration may have important implications for the Laceys' (1974) theory of sensorimotorcardiac interactions. Indeed, several experiments have reported differences in perceptual sensitivity as a function of respiratory phase, which would be in accordance with the Laceys' model (Flexman, Demaree, \& Simpson, 1974; Diekhoff, 1977). Unfortunately, there is little empirical evidence to support the notion of vagal gating. Hayes (Note 2) reports a study by Gautier (1972) which demonstrated that cardiac decelerations could be elicited only during expiration. However, the quantification of the ECR was fairly crude and also did not correct for differences in RSA. Thus, decelerative "responses" might have been confused with decelerative phase of RSA which usually occurs during expiration. Similarly, the work of Hart (1975) does not address this question, since accelerative responses were studied which were probably mediated by vagal inhibition rather than excitation. The present study, therefore, assessed any difference in RSA-corrected decelerative ECRs elicited by auditory stimuli presented at different phases of the breathing cycle.

In the present study, two independent groups of subjects were presented with a series of tones of moderate intensity at either inspiration or expiration.
It was predicted that if the replacement hypothesis concerning the summation of SA and ECRs was correct, then no differences in the ECR would be obtained between the two groups. However, if the summation hypothesis was correct, the ECR would differ as a function of respiratory phase. Furthermore, if a RSA correction procedure was employed, the difference between the two groups would be reduced. Any further differences between RSA-corrected ECRs for the two groups might reflect the possibility of vagal gating. More specifically, it was predicted that the decelerative response would be inhibited or greatly attenuated during inspiration.

In addition to the above prediction, the effects of respiratory phase upon skin conductance responses (SCRs) and breathing pattern were also assessed.

\section{Method}

\section{Subjects}

The subjects were 14 male and 14 female volunteers (age range 22-41 years) who were assigned randomly to one of two groups. Each group was composed of 7 males and 7 females. For the male subjects, the male author was the experimenter, and for the female subjects, the female author acted as the experimenter.

\section{Apparatus and Procedure}

Pulse-volume changes were recorded using an earlobe photoplethysmograph connected to a Grass dc preamplifier (Model 7P1A) and employing a time constant of $1 \mathrm{sec}$. The output from the driver amplifier was used to trigger an instantaneous rate-meter (Devizes 4522), which provided a measure of heart rate (HR) in beats per minute. This was then displayed using a Grass Model 7 polygraph and simultaneously recorded on magnetic tape using an FM tape recorder. The FM tape recorder was calibrated before each session, using a variable-frequency pulse generator.

Respiration was recorded using a thermistor placed in the subject's left naris. Changes in respiration were monitored as changes in dc level, using a Grass 7P1A preamplifier with PGR input setting. This signal was then displayed on the polygraph.

Skin resistance was recorded from the middle phalanges of the index finger and the middle finger of the left hand using bipolar $\mathrm{Ag} / \mathrm{AgCl}$ electrodes and " $\mathrm{KY}$ " electrode gel. The current was $10 \mu \mathrm{A}$. Recordings were monitored on the polygraph and stored on magnetic tape for subsequent analysis.

The subject was seated in a comfortable chair in a dimly illuminated room adjoining the equipment room, and the electrodes were attached. He was then instructed that, after a 3-min rest period, he would hear a series of tones, the intensity of which would not be unpleasant. Six tones were presented at randomly ordered intervals of 30,45 , and $60 \mathrm{sec}$ (mean interval $=45 \mathrm{sec}$ ). The tones were produced using an AMF wide-range oscillator and were delivered binaurally through AKG stereo headphones. The tone frequency was $1,000 \mathrm{~Hz}$ and the intensity was $75 \mathrm{~dB}$ (re .0002 dynes $/ \mathrm{cm}^{2}$ ) as assessed using a Dawes sound-level meter. Stimulus duration was $1 \mathrm{sec}$, and the rise time was instantaneous.

All subjects received an identical series of stimuli. However, one group of subjects received the stimuli only during midinspiration, whereas the other group of subjects received the stimuli only in midexpiration. To insure the accurate presentation of stimuli at the appropriate point in the respiration cycle, the actual onset of stimulus presentation was controlled using a Schmitt trigger operated via the respiration signal. Respiration recordings were centered around the $0-\mathrm{V}$ level of the chart record, and a trial was initiated by pressing a button before the ascending limb of the respiration signal reached this midline. The buttonpress energized a Schmitt trigger, which then operated the stimulus presentation 
equipment when the respiration signal crossed the $0-\mathrm{V}$ threshold. A marker was also recorded both on the chart record and on magnetic tape.

In addition to stimulus presentation, the Schmitt trigger was also used to mark a control or pseudostimulus trial about $20 \mathrm{sec}$ prior to each stimulus presentation. This control trial consisted only of a stimulus marker, since the tone generator was disconnected on these occasions. Control trials were obtained in order to derive RSA-corrected scores as described in the next section.

Following the series of tones, the subject rated the intensity of the series using a 5-point rating scale. The anchor points were: very loud (1), loud (2), neutral (3), soft (4), and very soft (5).

\section{Scoring}

HR was scored for $10 \mathrm{sec}$ poststimulus by sampling the output from the instantaneous rate meter at a digitizing rate of $50 \mathrm{~Hz}$ using a PDP-12 labratory computer. HR was also obtained for $10 \mathrm{sec}$ during a control period which always preceded stimulus onset. RSA-corrected difference scores were obtained in a manner similar to Hart's (1975) by subtracting the control periods from the experimental trials. An adjustment for differences in overall HR level was also made for each trial based on the difference between the first poststimulus beat for each set of control and experimental trials. This beat was chosen, since the use of a cardiotachometer introduced an average lag of one beat. Thus, the first poststimulus beat was effectively the interbeat interval at or prior to stimulus onset. The poststimulus values for control, experimental, and RSA-corrected difference scores were then averaged separately over the six stimulus presentations. Trials with gross respiratory irregularities were omitted from the analysis. Altogether, seven control and eight experimental trials were excluded. The averages were then converted to a second-by-second time base by evaluating the mean of the 20 -msec samples for each poststimulus second.

Skin resistance responses were evaluated as the difference between prestimulus level and peak level within $10 \mathrm{sec}$ following stimulus onset. These were then transformed into changes in log conductance.

Respiration rate was assessed by measuring the period of each ascending and descending limb of two consecutive respiratory cycles for both the control and the experimental trials. Difference scores were then obtained by subtracting the values for each control trial from the values of its corresponding experimental trial.

\section{Results}

\section{Cardiac Activity}

The data from the control, experimental, and corrected difference scores were analyzed separately using a repeated-measures ANOVA with respiratory phase ( 2 levels) and sex ( 2 levels) as the between-groups factors and poststimulus seconds (10 levels) as the repeated-measures factor. A significance level of .05 and conservative degrees of freedom were employed throughout the analysis. The averages of both the control and the experimental data revealed a pattern characteristic of respiratory sinus arrhythmia, which commenced either with acceleration or deceleration in accordance with the stimulus position within the respiratory cycle.

Analysis of the control trial averages resulted in a significant Respiratory Phase by Seconds interaction $[F(1,24)=11.18, p<.01]$. Trend analysis revealed significant interactions for the Respiratory Phase by Quadratic, Cubic, and Quartic Components $[\mathrm{F}(1,24)$

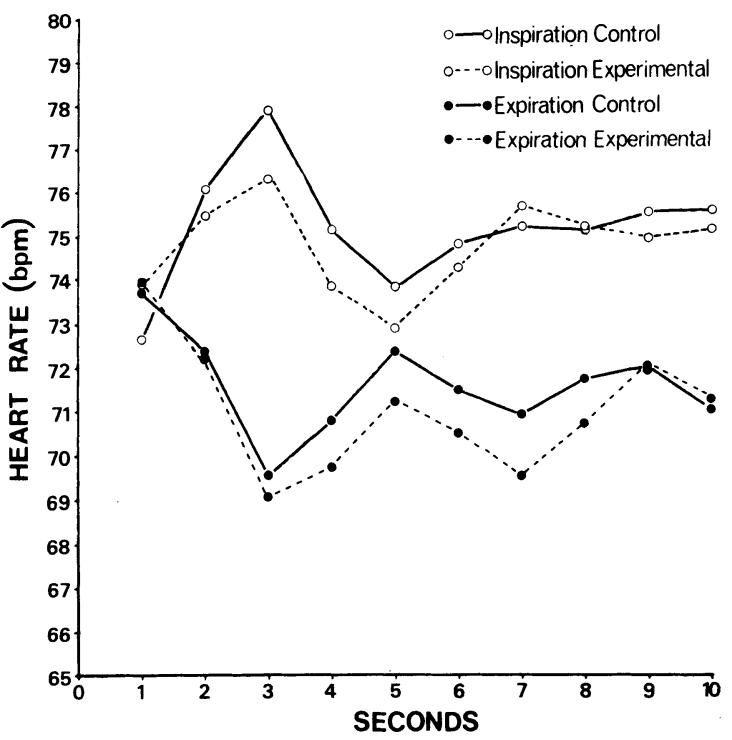

Figure 1. HR changes during control and experimental trials.

$=6.56(\mathrm{p}<.05), 35.31(\mathrm{p}<.01)$, and $23.64(\mathrm{p}<.01)$, respectively].

Analysis of the experimental trial averages resulted in a significant Respiratory Phase by Seconds interaction $[\mathrm{F}(1,24)=7.02, \mathrm{p}<.01]$. Trend analysis revealed significant interactions for the Respiratory Phase by Quadratic, Cubic, and Quartic components $[F(1,24)=10.32,6.31$, and 10.85 , respectively; ps < $.01]$. These results, together with the control trial data, are shown in Figure 1.

Analysis of the corrected difference scores resulted in no significant main effect or interactions when conservative degrees of freedom were employed. However, trend analysis across seconds revealed significant quadratic and cubic components $[F(1,24)=$ $13.57(\mathrm{p}<.01)$ and $5.96(\mathrm{p}<.05)$, respectively], a Respiratory Phase by Cubic Component interaction $[F(1,24)=9.64, p<.01]$, a Sex by Linear Component and a Sex by Quadratic Component interaction $[\mathrm{F}(1,24)=10.52(\mathrm{p}<.01)$ and $8.20(\mathrm{p}<.01)$, respectively], and a Respiratory Phase by Sex by Cubic Trend interaction $[F(1,24)=4.98, p<.05]$. Thus, females showed greater deceleration than males, and stimuli delivered during inspiration produced a greater initial deceleration than did those delivered during expiration. These data are displayed in Figure 2.

\section{Skin Conductance Responses}

Changes in log conductance were subjected to a repeated measures ANOVA with respiratory phase and sex as the between-group factors and trials (6 levels) as the repeated-measures factor. Only the trials effect was significant $[F(1,22)=24.23, p<.01]$, indicating that SCR amplitude decreased with repeated presentations. However, when nonconservative degrees of freedom were employed, significant inter- 


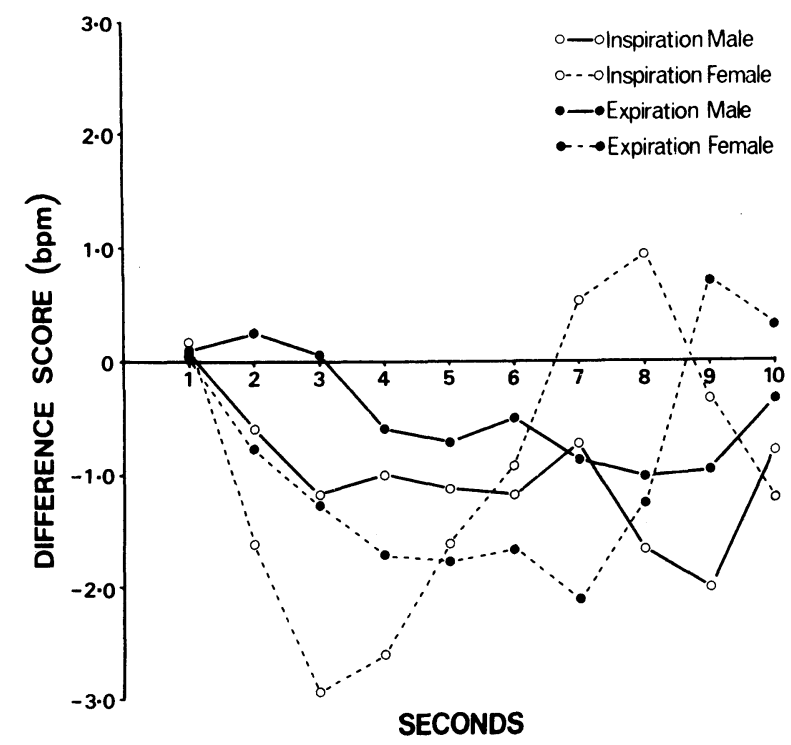

Figure 2. RSA-corrected difference scores following stimulus presentation.

actions were found for Respiratory Phase by Trials $[\mathrm{F}(5,101)=2.45, \mathrm{p}<.05]$ and Respiratory Phase by Sex by Trials $[F(5,101)=3.43, p<.01]$. Examination of the group means revealed smaller SCR amplitudes in the female expiration group. Since the use of nonconservative degrees of freedom has been questioned in designs of this type (Jennings \& Wood, 1976), these results should be interpreted with some caution.

\section{Respiration}

Difference scores derived from the control and experimental trials were subjected to a repeated measures ANOVA with respiratory phase and sex as the between-group factors and trials and periods (4 levels) as the repeated-measures factors. No significant effects were found when conservative degrees of freedom were employed. However, when using nonconservative degrees of freedom, both the trials main effect $[F(5,114)=3.31, p<.01]$ and the Trials by Respiratory Phase interaction $[\mathrm{F}(5,114)=3.96, \mathrm{p}<.01]$ were significant (see Footnote 2). Examination of the group means revealed an increase in respiration rate during the first two trials in the inspiration group. Once again, these findings should be interpreted cautiously since they employ the nonconservative test. There were no significant differences in the number of respiratory irregularities between the control and experimental trials.

\section{Cardiac-Respiratory Interactions}

Since it is possible that the ECR might be mediated by changes in respiratory activity, an attempt was made to establish the relationship between these two measures. The mean change in respiration rate was correlated with the mean amplitude of the decelerative response for each subject. Neither the individual within-group correlations nor the pooled within-group correlation $(\mathrm{r}=.16, \mathrm{df}=23)$ was significant.

\section{Ratings}

No significant differences were found between groups in the subjective rating of tone intensity $[F(1,22)$ $=2.62$ ] . For stimuli presented during expiration, the mean ratings for males and females were $3.29(\mathrm{SD}=$ $.49)$ and $4.00(\mathrm{SD}=.63)$, whereas for stimuli delivered during inspiration, the means for males and females were $3.83(\mathrm{SD}=.41)$ and $3.57(\mathrm{SD}=.53)$, respectively.

\section{DISCUSSION}

The results of this experiment clearly demonstrate the effect of RSA upon the ECR. The presence of RSA in prestimulus HR was shown by the difference in the control trials between the inspiration and expiration groups. The inspiration group was characterized by initial cardiac acceleration, whereas the expiration group displayed cardiac deceleration. This disparity can be attributed to differences in RSA originating from the point in the RSA cycle at which samples were obtained. The attenuation of the cardiac changes during the latter half of both samples was probably due to individual differences in RSA cycle length. The influence of RSA upon the ECR itself is clearly evident from examination of the experimental trials, since the direction of the evoked poststimulus cardiac activity differed between the two groups. In the absence of correction for RSA, these results might be interpreted as two distinct responses. Accordingly, correction for RSA resulted in a single response profile for the two groups consisting of cardiac deceleration, which has been identified as a component of the OR (Graham \& Clifton, 1966). Altogether, these results support the summation hypothesis regarding the interaction between RSA and the ECR to stimuli of moderate intensity.

The correction for RSA resulted in a reduction in the percentage sums of squares associated with the Respiratory Phase by Seconds interaction. For control and experimental trials, this term accounted for $29.2 \%$ and $18.7 \%$ of the within-subjects sums of squares. However, correction for RSA resulted in a reduction in this term to only $6.1 \%$ of the withinsubjects sums of squares. This reduction in variance is similar to the findings reported by Hart (1975) for accelerative responses. Thus, these results further support the use of SA or RSA correction procedures in the derivation of ECRs to simple stimuli.

It has been suggested that ECRs to simple stimuli may be mediated by changes in respiration. A number of studies have examined this claim. Steinschneider (1968) reported that respiratory responses to simple auditory stimuli did not predict the form of the ECR 
in neonates. Similarly, Klorman, Weissberg, and Wisenfeld (1977) found differences in the ECR to affective stimuli but no differences in respiration. Studies that have suggested respiratory-mediated ECRs have tended to report short-latency accelerative startle responses accompanied by inspiratory gasps (Graham \& Clifton, 1966; Smith \& Strawbridge, 1969). Hence, it would appear that decelerative responses are independent of respiratory changes. This conclusion is supported by the failure in the present study to demonstrate a significant correlation between cardiac deceleration and the change in respiratory cycle length.

Measures of respiration did not differ generally between either the experimental and control trials or the inspiration and expiration groups. However, there was some evidence of a decrease in the duration of the respiratory cycle following the presentation of the first two stimuli in the inspiration group. A similar increase in respiration rate has been previously reported by McCallum, Burch, and Roessler (1969), Porges and Raskin (1969), and Steinschneider (1968). In contrast, Barry (1977) has argued that a decrease in respiration rate follows brief simple auditory stimuli. However, Barry's study and those he cited mainly employed stimulus intensities around the threshold level. It is probable, therefore, that stimulus intensity is a critical factor in determining respiratory change to simple stimuli.

The final issue raised by these data concerns the notion of "vagal gating." It was predicted that decelerative ECRs would be inhibited or attenuated during inspiration. Although trend analysis did reveal differences in the ECR as a function of respiratory phase, they were not in the predicted direction. Larger decelerations were obtained for the inspiration rather than the expiration group. Thus, the "vagal gating" hypothesis was not supported by these data. However, there does appear to be a difference in the form of the ECR as a function of stimulus position in the breathing cycle. Several explanations can be suggested.

First, it could be argued that the disparity between the two groups arose due to the change in breathing cycle length in the inspiratory group. This could either lead to a respiratory mediated change in cardiac activity or a difference in the ECR as a result of a change in RSA phase (Barry, 1979). Both these explanations are unlikely, since no correlation was found between degree of cardiac deceleration and the change in respiratory cycle length. Furthermore, if a change in inspiration had mediated changes in cardiac activity, a decrease, rather than an increase, in the amount of cardiac deceleration might have been expected.

The second explanation reexamines the original "vagal gating" hypothesis. The differences in ECR may still be attributed to "vagal gating," if there had been a shift in the phase relationships between respiratory cycle, stimulus onset, and RSA. For instance, the phase relationship between respiration and RSA is dependent upon the breathing frequency (Angelone $\&$ Coulter, 1964). Similarly, the use of a nasal thermistor both to record respiration and control the position of stimulus onset may have introduced a delay of about $1 \mathrm{sec}$ into the system. These effects may have produced a difference in the phase relationship. However, examination of the control and experimental trials in Figure 1 indicate that cardiac activity was directly in phase with respiratory activity. Another possible effect of respiratory phase upon the "vagal gating" hypothesis concerns the latency of the ECR. Since stimuli were delivered during midinspiration and midexpiration, any interaction between vagal gating and the efferent mediation of the ECR may have occurred in the following phase of the respiratory cycle. Hence, stimuli delivered in midexpiration might be expected to produce smaller decelerations, since the gating effect on the ECR would occur in the subsequent inspiratory phase of the cycle.

In summary, these results stress the importance of respiration and RSA for the quantification of the ECR. As regards the "vagal gating" hypothesis, there would appear to be some difference between ECRs obtained at different phases of the respiratory cycle. However, this finding needs to be replicated, and the exact nature of the phase relationship among respiration, cardiac activity, and stimulus onset needs to be established before an adequate account of this phenomenon can be made.

\section{REFERENCE NOTES}

1. Kamath, S. Personal communication, 1977.

2. Hayes, R. W. Measuring heart-rate responses in psychophysiology. Paper presented at the 1st Annual Meeting of the Psychophysiology Society, London, 1973.

\section{REFERENCES}

Angelone, A., \& Coulter, N. A. Respiratory sinus arrhythmia: A frequency dependent phenomenon. Journal of Applied Physiology, 1964, 19, 479-482.

BARRY, R. J. Failure to find evidence of the unitary OR concept with indifferent low-intensity auditory stimuli. Physiological Psychology, 1977, 5, 89-96.

BARRY, R. J. Correction for sinus arrhythmia in the evoked cardiac response: A timebase problem. Biological Psychology, 1979, 9, 221-224.

Berg, R. L., \& Beebe-Center, J. G. Cardiac startle in man. Journal of Experimental Psychology, 1941, 28, 262-279.

Davidson, N. S., Goldner, S., \& McCloskey, D. I. Respiratory modulation of baroreceptor and chemoreceptor reflexes affecting heart rate and cardiac vagal efferent nerve activity. Journal of Physiology, 1976, 259, 523-530.

DieкноғF, G. M. Effects of phase-of-respiration on GSR detection. British Journal of Psychology, 1977, 68, 499-502.

Flexman, J. E., Demaree, R. G., \& Simpson, D. D. Respiratory phase and visual signal detection. Perception \& Psychophysics, 1974, 16, 337-339.

GaUtie R, H. Respiratory and heart rate responses to auditory stimulations. Physiology \& Behavior, 1972, 8, 327-332.

Graham, F. K., \& Clifton, R. K. Heart rate change as a com- 
ponent of the orienting response. Psychological Bulletin, 1966, 65, 305-320.

HART, J. D. Cardiac response to simple stimuli as a function of the respiratory cycle. Psychophysiology, 1975, 12, 634-636.

Iriuchijima, J., \& Kumada, M. Activity of single vagal fibres efferent to the heart. Japanese Journal of Physiology, 1964, 14, 479-487.

Jennings, J. R., \& Wood, C. C. The $\varepsilon$-adjustment procedure for repeated-measures analyses of variance. Psychophysiology, 1976, 13, 277-278.

Katona, P. G., Poitras, J. W., Barnett, G. O., \& Terry, B. S. Cardiac vagal efferent activity and heart period in the carotid sinus reflex. American Journal of Physiology, 1970, 218, 1030-1037.

Klorman, R., Weissberg, R. P., \& Wiesenfeld, A. R. Individual differences in fear and autonomic reactions to affective stimulation. Psychophysiology, 1977, 14, 45-51.

LACEY, B. C., \& LACEY, J. I. Studies of heart rate and other bodily processes in sensorimotor behavior. In P. A. Obrist, A. H. Black, J. Brener, \& L. V. Dicara (Eds.), Cardiovascular psychophysiology: Current issues in response mechanisms, biofeedback and methodology. Chicago: Aldine, 1974.

Lobstein, R., Webs, R., \& CoRT, J. Background noise levels and heart rate orienting: Response detection using time series analysis. Psychophysiology, 1978, 15, 316-319.

McCallum, M., Burch, N. R., \& Roessler, R. Personality and respiratory responses to sound and light. Psychophysiology, 1969, 6, 291-300.

Melcher, A. Respiratory sinus arrhythmia in man. Acta Physiologica Scandinavica, 1976, Supplement 435.
Obrist, P. A., Wood, D. M., \& Perez-Reyes, M. Heart rate during conditioning in humans: Effects of UCS intensity, vagal blockade, and adrenergic block of vasomotor activity. Journal of Experimental Psychology, 1965, 70, 32-42.

Porges, S. W., \& RAskin, D. C. Respiratory and heart rate components of attention. Journal of Experimental Psychology, 1969, 81, 497-503.

Smith, D. B. D., \& Strawbridge, P. J. The heart rate response to a brief auditory and visual stimulus. Psychophysiology, 1969, 6, 317-329.

STEINSCHNEIDER, A. Sound intensity and respiratory responses in the neonate. Psychosomatic Medicine, 1968, 30, 534-541.

Turpin, G., Lobstein, T., \& Siddee, D. A. T. Phasic activity: The influence of prestimulus variability. In I. Martin \& P. H. Venables (Eds.), Techniques in psychophysiology. London: Wiley, 1980.

Turpin, G., \& Siddle, D. A. T. Measurement of the evoked cardiac response: The problem of prestimulus variability. Biological Psychology, 1978, 6, 127-138.

\section{NOTES}

1. RSA specifically refers to cardiac variability associated with respiratory activity. SA is used to describe generally cardiac variability of a cyclic nature.

2. Degrees of freedom adjusted for missing values.

(Received for publication April 10, 1980; revision accepted June 17,1980 .) 